\title{
Revealing the Essence of Electric Permittivity Constant
}

\author{
Nader Butto \\ Dgania, Petah Tikva, Israel \\ Email: nader.butto@gmail.com
}

How to cite this paper: Butto, N. (2021) Revealing the Essence of Electric Permittivity Constant. Journal of High Energy Physics, Gravitation and Cosmology, 7, 210-217. https://doi.org/10.4236/jhepgc.2021.71011

Received: October 1, 2020

Accepted: January 15, 2021

Published: January 18, 2021

Copyright $\odot 2021$ by author(s) and Scientific Research Publishing Inc. This work is licensed under the Creative Commons Attribution International License (CC BY 4.0).

http://creativecommons.org/licenses/by/4.0/

\begin{abstract}
Electric constant believed fundamental constant determined only by physical measurement and cannot be calculated. A new theory about the origin and the essence of the electric constant is proposed and mathematical formulation is described. The vacuum is considered to be superfluid and the electric constant is described as a physical property of the "vacuum". Hydrodynamics laws are applied to calculate the elasticity and the compressibility of the vacuum to obtain the electric constant value. Thus, electric permittivity is the expression of compressibility of the vacuum which is the capability of the vacuum to permit electric field lines. In conclusion, electric constant is not fundamental constant but observable parameter of the vacuum which depends mainly by the vacuum density. This result could have important consequences in our understanding the origin of physical forces forward Universal Unified Theory based on one constant only, the density of the vacuum.
\end{abstract}

\section{Keywords}

Electric Constant, Elasticity, Vacuum Compressibility, Vacuum Density

\section{Introduction}

A physical constant is a physical quantity that generally believed to be both universal in nature and constant in time. The term fundamental physical constant is generally sometime used to refer to universal but dimensioned physical constants such as the speed of light in vacuum $\mathcal{c}$, magnetic permeability constant $\mu_{0}$ the gravitational constant $G$, Planck's constant $h$, the electric constant $\varepsilon_{0}$, and the elementary electron charge $e$ [1].

In electromagnetism, the free space has a resistance to the formation or propagation of the photons of the magnetic field. The photon is an electromagnetic vibration presented as alternating electric and magnetic field propagation 
in empty space (vacuum) at the speed of light.

The classical behavior of the electromagnetic field described by Maxwell's equations, which predict that the speed of light $c$ with which electromagnetic waves (such as light) propagate through the vacuum is related to the electric constant $\varepsilon_{0}$ and the magnetic constant $\mu_{0}$ by the equation

$$
c=1 / \sqrt{\varepsilon_{0} \mu_{0}} \text {. }
$$

According to this equation, the speed of the propagation of electromagnetic radiation, $c$, turns out to be dependent and inversely related to the values of the permittivity and the permeability of the medium. The vacuum permeability $\mu_{0}$, the vacuum permittivity $\varepsilon_{0}$, and the speed of light in vacuum $\mathrm{c}$ are widely considered as being fundamental constants and their values, escaping any physical explanation, are commonly assumed to be invariant in space and time.

A physical quantity indicated by a physical constant does not depend on the unit system used to express the quantity; however, the numerical values of dimensional physical constants depend on the units used to express them. Therefore, the numerical values of these constants are not fundamental.

The ultimate physical origin of electric constant, $\varepsilon_{0}$, could provide important insights into this understanding.

In previous articles the nature and the origin of the fine structure constant, [2] the gravitational constant $G$, [3] and magnetic constant $\mu_{0}$ [4] were described.

In this paper, the origin and essence of electric constant are described. The analysis based on the following assumptions:

1) Vacuum filled with energy that has measurable density.

2) Hydrodynamic laws are applicable to superfluid vacuum.

3) The density of the vacuum is the only basic property that governs the compressibility and speed of light through the vacuum.

4) The compressibility is conserved momentum which depends on the speed of light and density of the vacuum.

\section{The Density of the Superfluid Vacuum}

Despite the fact that the theory of quantum mechanics not predicted upon any property of space at all, the space ideas frequently used to justify mathematical procedures and this imply amount of detail space properties like the speed of light in the vacuum governed by the vacuum permeability and permittivity.

A vacuum by definition has no mass, since there is nothing in it to produce mass. However, during the early years of quantum mechanics, Paul Dirac theorized that the vacuum was actually filled with particles in negative energy states [5] giving rise to the concept of the "physical vacuum" which is not empty at all. Quantum mechanics also predicted that invisible particles could become materialized for a short time and that these virtual particle appearances should exert a force that is measurable. Thus, the vacuum's totally empty space is actually a seething turmoil of creation and annihilation, which to the ordinary world appears calm because the scale of fluctuations in the vacuum is tiny and the fluc- 
tuations tend to cancel each other out. Quantum fluctuation is defined as "the temporary change in the amount of energy in a point in space" [6].

The concept of zero-point energy was developed in Germany by Albert Einstein and Otto Stern in 1913, using a formula developed by Max Planck in 1900 [7].

"Zero-point energy" refers to the energy of the system at temperature $\mathrm{T}=0$, or the lowest quantized energy level of a quantum mechanical system, so where there should be no heat or any other form of energy remaining but it has measurable effects. The effects of vacuum energy can be experimentally observed in various phenomena such as spontaneous emission, the Casimir effect, the Van der Waals bonds and the Lamb shift [8].

In quantum electrodynamics QED, vacuum is a state with no matter particles, and no photons contains vacuum fluctuations and a finite energy called vacuum energy, this vacuum is referred to as a state where all fields are in their unexcited ground-state, with no matter particles, and also no photons, no gravitons etc., however, when we put a particle in it, it feels a force.

It is the quantum state with the lowest possible energy which behaves as superfluid. The Superfluid is characterized behave like a fluid without or with extremely low viscosity and with extremely high thermal conductivity. It is a perfect fluid in the sense that it is non-particulate. It has no structural memory. Once changed, it has no tendency to go back to its former condition.

Superfluid vacuum theory proposes the mass generation mechanism which may replace or supplement the electroweak Higgs one. It was shown that masses of elementary particles can arise as a result of interaction with the superfluid vacuum, similarly to the gap generation mechanism in superconductors [9] [10].

Super fluidity of the vacuum is the basis for Maxwell's equations, special relativity, and general relativity.

Maxwell's equations united and mathematically quantified the interaction of electrical and magnetic effects and they explicitly developed as fluid dynamical models, and require an underlying physical medium.

Special relativity derived from Maxwell's equations. Einstein clearly realized that both special and general relativity based on fluid dynamical models [11].

Even though there is no consensus on the value of the density of the vacuum, vacuum energy density generally viewed as a fundamental property of the cosmos whose magnitude is the same value whether it is assessed in subatomic, astronomical or cosmological methods. The energy of the cosmological vacuum thought to influence the behavior of the Universe on cosmological scales and in the quantum realm influence the particles behavior.

The value of vacuum density, rely mainly on general relativity and been measured through astronomical observations that determine the curvature of space-time and the expansion of the universe.

According to physical cosmology, astronomy and celestial mechanics, the universe is permeated with a hypothetical form of energy called Dark energy that 
full all of space and tends to accelerate the expansion of the universe [12].

The measurement of universal expansion based on the relation between galaxy velocity $(v)$ and its distance $(d)[13]$

$$
v=H_{0} \times d .
$$

where $H_{0}$ is Hubble constant. This relation is the well-known Hubble Law. It indicates a constant expansion of the cosmos where, galaxies, recede from each other at a constant speed per unit distance; thus, more distant objects move faster than nearby ones.

It is important to note that the study of the expansion rate of the universe has shown that the universe is close to critical density. Critical density is the value at which the Universe is balanced and expansion is halted.

The density is typically expressed as a fraction of the density required for the critical condition to be fulfilled through the use of a parameter known as omega $(\Omega)$ where $\Omega=\rho / \rho_{\text {critical }}$. The limiting critical density is described by $\Omega=1$. Currently, the estimated sum of the contributions to the total density parameter, $\Omega_{0}$, is $\Omega_{0}=1.02 \pm 0.02$ which indicates that the universe is close to critical density.

Hence, the critical density that defines the boundary condition between the open and closed solutions of the standard cosmological model which it can be calculated by the formula [14]

$$
\rho_{c r}=3 H_{0}^{2} / 8 \pi G
$$

where $\rho_{\mathrm{cr}}$ is the critical density, $\mathrm{H}_{0}$ is the current value of the Hubble constant and $G$ the universal gravity constant $(6.67384 \pm 0.00080) \times 10^{-11} \mathrm{~m}^{3} / \mathrm{kg} \cdot \mathrm{s}$ or $\mathrm{Nm}^{2} / \mathrm{kg}^{2}$.

The most recent result [15], indicates that the value of Hubble constant is $H_{0}$ $=71.9+2.4-3.0 \mathrm{kms}^{-1} \mathrm{Mpc}^{-1}$, if the upper limit of uncertainty is taken, the Hubble constant value is

$$
H_{0}=\mathrm{km} / \mathrm{s} / \mathrm{Mpc}=2.29 \times 10^{-18} \mathrm{~s}^{-1}
$$

where the number of $\mathrm{km}$ in an Mpc is $3.09 \times 10^{22} \mathrm{~m}$

Considering that the inertial mass of the Observable Universe is

$$
M_{U}=c^{3} / 2 H_{0} G
$$

Then the $M_{U}=0.8810705493 \times 10^{56} \mathrm{~kg}$.

And the volume of the universe is

$$
V_{u}=4 / 3 \pi R_{u}^{3}=4 / 3 \pi\left(c / H_{0}\right)^{3}=8.9364367479 \times 10^{81} \mathrm{~m}^{3}
$$

where $R$ is the radius of the Universe.

Then the cosmological density $\rho_{c o}$ is calculated to be:

$$
M_{U} / V_{U}=\rho_{c o}=9.85930493 \times 10^{-27} \mathrm{Kg} / \mathrm{m}^{3}
$$

\section{The Elasticity of the Vacuum}

Magnetic and electric constants are dimensional parameters of great significance in characterizing the physical properties of the vacuum. Fundamental properties 
of this vacuum or empty space given by these two parameters, electric permittivity and the speed of light in vacuum.

The physical constant $\varepsilon_{0}$, commonly called the vacuum permittivity, is the value of the absolute dielectric permittivity of classical vacuum. It is the capability of the vacuum to permit the propagation of electric field lines in space.

Likewise, $\varepsilon_{0}$ appears in Maxwell's equations, which describe the properties of electric and magnetic fields and electromagnetic radiation, and relate them to their sources.

Furthermore, permittivity is a vacuum property that affects the force between two point charges in the material. For example, the force between two separated electric charges (in vacuum) is given by Coulomb's law:

$$
F_{c}=q_{1} q_{2} / 4 \pi \varepsilon_{0} r^{2}
$$

where $q_{1}$ and $q_{2}$ are the charges, and $r$ is the distance between them.

The value of vacuum permittivity $\varepsilon_{0}$ currently defined by the formula:

$$
\varepsilon_{0}=1 / \mu_{0} c^{2}
$$

where $c$ is the defined value for the speed of light in classical vacuum and $\mu_{0}$ is the magnetic permeability.

In SI units the value of $\varepsilon_{0}$ is

$$
\varepsilon_{0}=8.854187817 \ldots \times 10^{-12} \mathrm{~F} / \mathrm{m} \text { (farads per metre) } .
$$

This constant relates the units for electric charge to mechanical quantities such as length and force.

Since photons carry linear momenta their interactions among themselves or with other quantum fields of space-time, matter, or energy can be described properly by a quantum theory of elasticity.

Classical elasticity defined as the property of a substance by which it tends to restore its shape or size after being subjected to deforming stresses.

In hydrodynamics, the elasticity of the medium depends on its density. The velocity of particle in an elastic medium expressed by the formula:

$$
v=(E / d)^{1 / 2}
$$

WHERE $\mathrm{v}=\mathrm{c}$ light velocity, E elasticity and $d=\rho$ density of the medium, indicating that the velocity of the particle is directly related to the elasticity of the medium and inversely related to its density.

Therefore, the elasticity of the vacuum calculated to be:

$$
E=\rho c^{2}
$$

This is a momentum equation and momentum neither created nor destroyed, but only changed through the action of forces as described by Newton's laws of motion, therefore it is constant.

The elasticity of the vacuum could be calculated according to Equation (8)

Where $\rho=9.85930493 \times 10^{-27} \mathrm{~kg} / \mathrm{m}^{3}$ and $\mathrm{c}^{2}=9 \times 10^{16} \mathrm{~m}^{2} / \mathrm{s}^{2}$

$$
E=\rho c^{2}=8.87337441 \times 10^{-10} \mathrm{kgm} / \mathrm{s}^{2} \cdot \mathrm{m}^{2} \text { or }=\mathrm{N} / \mathrm{sm}^{2}
$$


This is an expression of force exercised on substance that passes through a given surface area per unit of time.

However, the vacuum is displaced by the substance have a volume therefore, elasticity of the vacuum should take in consideration the volume and not only the surface.

Therefore, dividing this value in one unite of length, $\mathrm{m}$, to obtain the force needed to move volume of vacuum per unit of time:

Thus: $E=8.87337441 \times 10^{-10} \mathrm{kgm} / \mathrm{s}^{2} \cdot \mathrm{m}^{3}$ or $\mathrm{kg} / \mathrm{s}^{2} \cdot \mathrm{m}^{2}$

The bulk modulus describes volumetric elasticity, or the tendency of an object to deform in all directions when uniformly loaded in all directions; it defined as volumetric stress over volumetric strain under uniform compression, and is the inverse of compressibility.

An elastic modulus has the form:

$$
\lambda=\text { stress/volume strain }
$$

where, stress is the force causing the deformation divided by the area to which the force is applied.

The Modulus of Elasticity is a number that measures an object or substance's resistance to being deformed elastically when a force is applied to it. The elastic modulus of an object defined as the slope of its stress-strain curve in the elastic deformation region [16].

If stress is measured in pascals, and strain is a dimensionless quantity, thus the units of $\lambda$ will be pascals as well [16]. Pascal units are kilogram per square centimeter $\left(\mathrm{kg} / \mathrm{cm}^{2}\right)$, equal to the pressure of one newton per square meter.

Converting Equation (8) to Pascal units we obtain the same value but different than electric constant but with inverse units.

$$
E=8.87337441 \times 10^{-12} \mathrm{kgcm} / \mathrm{s}^{2} \cdot \mathrm{cm}^{3} \text { or } \mathrm{P} / \mathrm{s}^{2}
$$

Normally in a liquid a force applied uniformly over the surface of an object will compress it uniformly and reduces the volume. The stress in this case is simply described as a pressure $(P=F / A)$. The resulting volume strain is measured by the fractional change in volume $\left(\theta=\Delta V / V_{0}\right)$. In this case the compressed volume is smaller than the original one, therefore $\Delta V<V_{0}$. However, the passage of a substance in a liquid exercise pressure over the liquid and expand it, such the passage of electric field in the vacuum, thus the compressed volume is bigger than the original one, therefore $V_{0}<\Delta V$, which gives reciprocal fractional change in volume.

The reciprocal of bulk modulus is called compressibility, its symbol is usually $\beta$ (beta) which can be expressed as: $\beta=1 / \rho c^{2}$.

The SI unit of compressibility is the inverse pascal $\left[\mathrm{Pa}^{-1}\right]$.

This formula indicates that the compressibility of the vacuum is inversely related to velocity of the particle in the vacuum and to the density of the vacuum. When body travel in a liquid it is under shear stress that means pressure on it. The units of shear stress are newton per area, while the units of expandability of 
the medium are reciprocal newton times area, the same units of electric permittivity.

This would make sense in terms of dimensions since elasticity modulus is Newton $\times \mathrm{m}^{-2}$ whilst permittivity Newtons ${ }^{-1} \times \mathrm{m}^{-2}\left(\mathrm{C}^{2} / \mathrm{N} \cdot \mathrm{m}^{2}\right)$.

Therefore, electric permittivity is the expression of compressibility of the vacuum which is the capability of the vacuum to permit electric field lines.

\section{Conclusions}

A new theory is proposed that can interpret the essence and the origin of the electric constant. Electric constant is related to the structure and properties of physical vacuum, which is considered as a superfluid medium characterized by specific properties such as density, compressibility, and speed.

In response to a small, rapidly applied and removed strain, the fluids may deform and then return to their original shape. The difficulty of the vacuum to expand, known as compressibility, a sort of difficulty of the atoms to form dipoles and polarization in the vacuum. The compressibility is inversely related to the density of the vacuum and the speed of light which are conserved quantity of linear momentum. Applying the laws of hydrodynamics to calculate the compressibility of the vacuum gives the same value of electric constant.

The result strongly suggests that electric constant is not a fundamental constant but it is an expression of the compressibility of the vacuum.

Therefore, vacuum density must be the only thing constant because there is nothing for it to depend on.

This study presents a new approach to determine the nature and the essence of fundamental constants in nature and opens a new perspective forward to a new theory of physics-based on one single constant which is the density of the vacuum. The origin and essence of the other fundamental constants such as Planck's constant $h$, and the elementary charge $e$ will be presented in separated papers.

These results could have the basis to change our understanding of physical laws and to formulate a new theory that unifies all physical forces forward Universal Unified Theory based on one constant only, the density of the vacuum.

\section{Limitation}

The calculated value of compressibility and thus the electric constant is in the range of uncertainty of the vacuum density that needs to be confirmed experimentally.

\section{Acknowledgments}

The author would like to thank Enago (http://www.enago.com/) for the English language and peer reviewer's review.

\section{Conflicts of Interest}

The author declares no conflicts of interest regarding the publication of this paper. 


\section{References}

[1] Panofsky, W.K.H. and Phillips, M. (1962) Classical Electricity and Magnetism. Addison-Wesley, Boston, 182.

[2] Butto, N. (2020) A New Theory on the Origin and Nature of the Fine Structure Constant. Journal of High Energy Physics, Gravitation and Cosmology, 6, 579-589. https://doi.org/10.4236/jhepgc.2020.64039

[3] Butto, N. (2020) New Mechanism and Analytical Formula for Understanding the Gravity Constant G. Journal of High Energy Physics, Gravitation and Cosmology, 6, 357-367. https://doi.org/10.4236/jhepgc.2020.63029

[4] Butto, N. (2020) The Essence and Origin of the Magnetic Constant. Journal of High Energy Physics, Gravitation and Cosmology, 6, 662-669. https://doi.org/10.4236/jhepgc.2020.64045

[5] Dirac, P.A.M. (1930) A Theory of Electrons and Protons. Proceedings of the Royal Society of London. Series A, 126, 360.

[6] Browne, M.W. (1990) New Direction in Physics: Back in Time. The New York Times.

http://www.nytimes.com/1990/08/21/science/new-direction-in-physics-bac k-in-time.html?pagewanted=all

[7] Laidler, K.J. (2001) The World of Physical Chemistry. Oxford University Press, Oxford, 324 .

[8] Barrow, J.D. (2000) The Book of Nothing: Vacuums, Voids, and the Latest Ideas about the Origins of the Universe (1st American ed.). Pantheon Books, New York.

[9] Zloshchastiev, K.G. (2011) Spontaneous Symmetry Breaking and Mass Generation as Built-In Phenomena in Logarithmic Nonlinear Quantum Theory. Acta Physica Polonica B, 42, 261-292. https://doi.org/10.5506/APhysPolB.42.261

[10] Avdeenkov, A.V. and Zloshchastiev, K.G. (2011) Quantum Bose Liquids with Logarithmic Nonlinearity: Self-Sustainability and Emergence of Spatial Extent. Journal of Physics B: Atomic, Molecular and Optical Physics, 44, Article ID: 195303. https://doi.org/10.1088/0953-4075/44/19/195303

[11] Condon, E.U. and Odishaw, H. (1958) Handbook of Physics, Section 29. McgrawHill, New York, 2-50.

[12] Peebles, P.J.E. and Ratra, B. (2003) The Cosmological Constant and Dark Energy. Reviews of Modern Physics, 75, 559-606. https://doi.org/10.1103/RevModPhys.75.559

[13] Hubble, E. (1929) A Relation between Distance and Radial Velocity among Extra-Galactic Nebulae. Proceedings of the National Academy of Sciences of the United States of America, 15, 168-173. https://doi.org/10.1073/pnas.15.3.168

[14] Oldershaw, R.L. (1987) The Self-Similar Cosmological Paradigm: A New Test and Two New Predictions. The Astrophysical Journal, 322, 34-36. https://doi.org/10.1086/165699

[15] Peebles, P.J.E. and Ratra, B. (2003) The Cosmological Constant and Dark Energy. Reviews of Modern Physics, 75, 559-606. https://doi.org/10.1103/RevModPhys.75.559

[16] Askeland, D.R. and Phulé, P.P. (2006) The Science and Engineering of Materials. 5th Edition, Cengage Learning, Boston, 198. 